\title{
Hints of exo-Earths spark desire for a closer look
}

\section{Of the latest clutch reported, one is among the most Earth-like yet, another orbits two suns.}

\section{BY ERIC HAND}

$\mathrm{G}^{\mathrm{s}}$ eoffrey Marcy finds it strange to turn people away from a conference about planets beyond our Solar System. "This is a field that had three of us in 1995," marvels Marcy, a pioneering exoplanet hunter at the University of California, Berkeley.

But as an organizer of last week's Extreme Solar Systems II conference, he had to decline applicants by the dozen. The Jackson Lake Lodge auditorium in Wyoming's Grand Teton National Park was packed to its 330-person capacity as speakers announced a flood of new detections, and the air was alive with talk of a 'golden age' of exoplanet astronomy.

Along with the discoveries came some sobering news. Rocky, Earth-like planets may be less common than many hoped, and unexpectedly 'noisy' stars are slowing the hunt. Moreover, astronomers cannot learn much beyond the basics - mass or size and orbit of the planets they do find. "What we need is a telescope in space that can image and take spectra of truly Earth-like planets," Marcy says. "We still need that desperately."

For now, however, indirect methods are keeping astronomers busy. One trove of discoveries came from a European team that watches stars for the slight wobble that signals the gravitational pull of an unseen planet. Their instrument, the High Accuracy Radial velocity Planet Searcher (HARPS), which is attached to a 3.6-metre telescope at the European Southern Observatory in La Silla, Chile, yielded 41 new planets, including one of the most Earth-like yet. At 3.6 times the mass of Earth, it sits in the 'habitable zone' around its star, the Goldilocks range of distances at which a planet's surface would be neither too hot nor too cold for water to be liquid.

There was also news from astronomers working with Kepler, a NASA space telescope that stares fixedly at a field of about 155,000 stars in search of transits: the very slight dip in the brightness of a star as a planet crosses in front. The Kepler team announced that they have now detected 1,781 candidate planets, including 123 that are Earth-sized (see 'Sizing up the sample'). Among the objects was a novelty: a circumbinary, or a planet orbiting a pair of stars.

Both groups are now confident enough to start making pronouncements about the

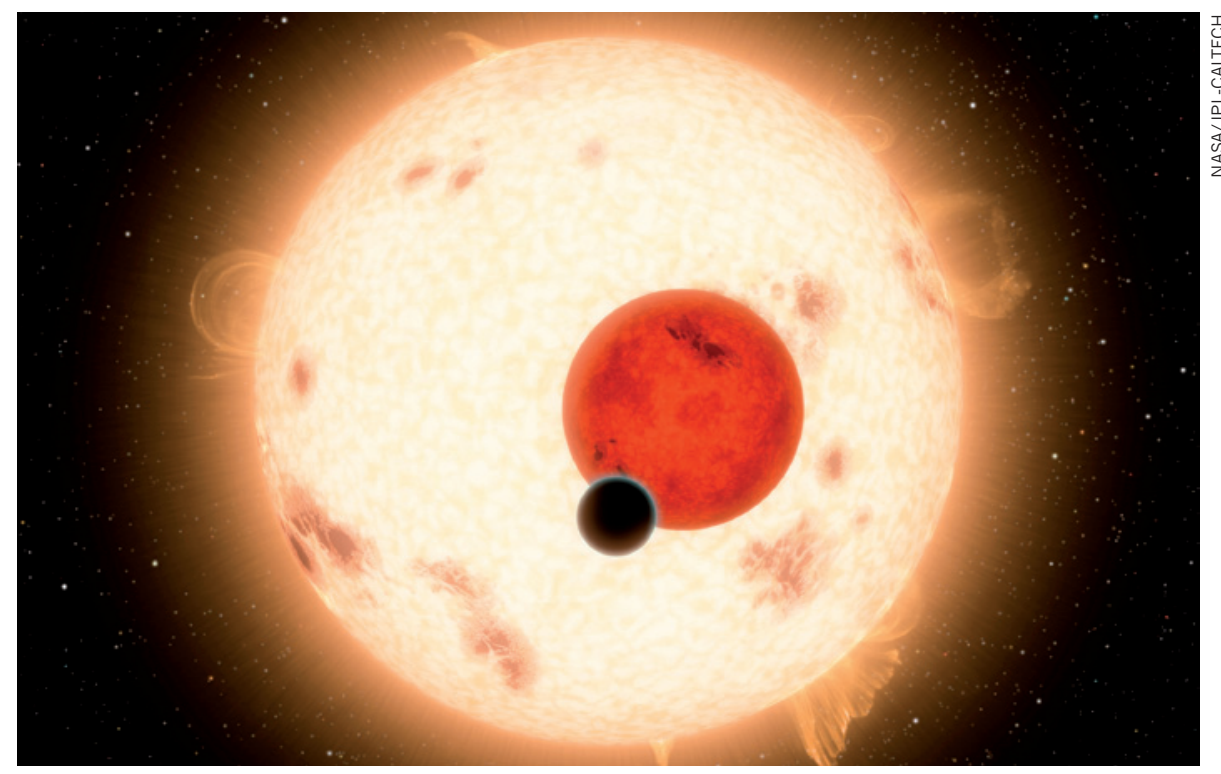

The Saturn-sized planet Kepler-16b (black) orbits two dwarf stars. It is not thought to be habitable.

statistics of planets in orbit close to a star the kind that both Kepler and HARPS are most sensitive to. The HARPS team, for instance, estimates that about half of Sun-like stars have at least one planet with an orbital period of 100 days or fewer, and that many of these systems boast several such planets. Greg Laughlin, an astronomer at the University of California, Santa Cruz, points out that the Solar System in which only Mercury has such a short period (88 days) - might end up looking like the oddball. "The big news is that there's this staggering population of planets that you'd never suspect from looking at our own Solar System," he says.

Extrapolating from Kepler data, Wesley Traub, chief scientist of NASA's exoplanet exploration programme, took a stab at a more fundamental question: what fraction of Sunlike stars have rocky, Earth-sized planets farther from the star, in a habitable zone? His optimistic answer: $34 \%$.

But many observers dismiss these calculations as premature. Marcy points out that the Kepler statistics suggest a drop off

\section{$\rightarrow$ NATURE.COM}

For more on the problem of stellar noise, see: go.nature.com/joxl6f in the frequency of planets smaller than 'super-Earths' - exoplanets with a radius 1.25-2 times that of Earth. "Is Kepler having trouble detecting truly Earth-sized planets?" asks Marcy. "Or are they rare? We don't know."

The stars themselves are making the hunt difficult. Instruments such as HARPS detect the tug of a planet from tiny shifts in the frequency of a star's spectral lines. Many researchers hoped that a new tool for calibrating the position of those lines, called a laser frequency comb, would enable HARPS to detect the minuscule signal from a planet as small as Earth. But it seems that all but the most quiescent stars have enough surface turbulence to drown out so small a signal. Similarly, the Kepler team has said that because of stellar noise, the mission will now need 8 years, rather than the originally planned 3.5 years, to detect all of the Earth-like planets around its target stars (see Nature 477, 142-143; 2011).

Even after true Earth analogues have been detected, neither HARPS nor Kepler will be able to determine whether they have an Earthlike atmosphere containing, for instance, oxygen or carbon dioxide. For a few giant planets that pass in front of their stars, instruments such as the Spitzer Space Telescope have been able to analyse the spectrum of starlight shining through the planet's thick atmosphere, gleaning clues about its composition. But Marcy says that doing transmission spectroscopy on the thin ring of atmosphere 
- surrounding an Earth-sized planet is beyond the reach of even the 6.5-metre James Webb Space Telescope, the orbital observatory that NASA hopes to launch by 2018 if it does not fall victim to cost overruns. "James Webb, yea or nay, is not the answer to our prayers," Marcy says.

As a result, researchers such as Marcy and Traub stress the need to go beyond the indirect techniques of HARPS and Kepler, and gather the faint light from the planet itself, which is normally invisible in the glare of the parent star. For that, astronomers need either a giant space telescope equipped with a device for blocking starlight, or an interferometer, consisting of several telescopes flying in formation. NASA did develop a proposal for such a space telescope, called Terrestrial Planet Finder, and the European Space Agency hoped to fly a similar mission called Darwin. But budgetary constraints have left both missions in limbo, unlikely to advance to the front of either agency's queue until well into the next decade. At the conference, Traub raised the issue. "People are not thinking deeply about the distant future. People are wrapped up with what they're doing right now," he says. "Clearly, I'm concerned."

But Laughlin isn't as worried. He says that the enthusiasm and momentum in planethunting may lead to an unexpected solution. "People have a history of being inventive when they need to be," he says. He points to French philosopher Auguste Comte, who in 1835 wrote that astronomers might be able to learn about the shapes, sizes and motions of stars, but that stellar densities, temperatures and chemical compositions would be "forever denied to us".

Within three decades, astronomical spectroscopy was starting to answer all of those questions.

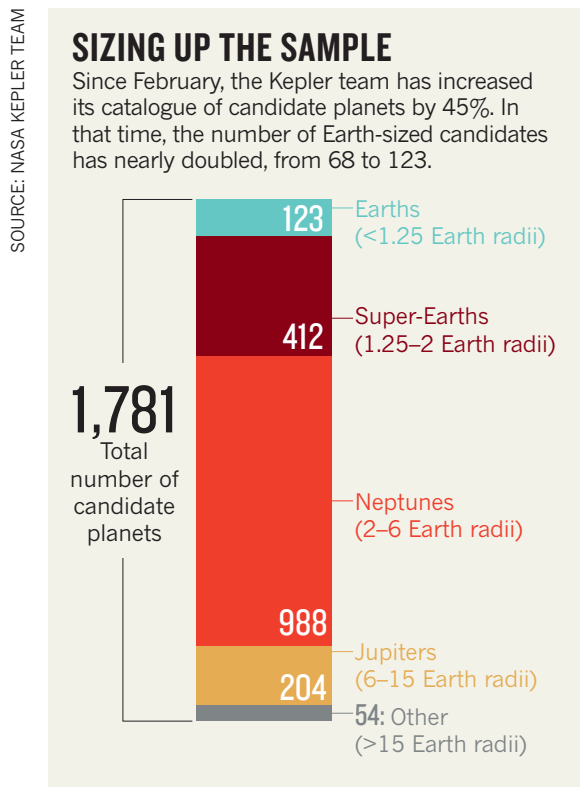

MISCONDUCT

Austria reinstates
disgraced doctor

Physician at heart of retracted clinical trial can return to work.

BY ALISON ABBOTT

$\mathrm{H}$ e carried out clinical trials without ethical approval. He failed to provide raw data for his high-profile publications. He falsified legal documents. But despite this record, an employment commission has ordered that Austrian urologist Hannes Strasser be readmitted to his teaching post at the Medical University of Innsbruck.

Now the university is trying to find a way out of the embarrassing situation - one that highlights the weakness and tardiness of Austria's system for dealing with research misconduct. "We are being forced by a legal decision to let him back when we think he has no place here," says the university's rector, Herbert Lochs.

The university suspended Strasser three years ago after serious concerns were first raised about his trial of a novel stem-cell therapy for urinary incontinence. The therapy relied on injecting stem cells and fibroblasts derived from the patients' own tissue into the urinary sphincter; it had been developed by Innovacell Biotechnologie in Innsbruck, a company cofounded by Strasser, and with which he is no longer involved. But many patients reported no improvement after the therapy, and others claim that it caused their bladders to seal over.

A subsequent investigation by the Austrian government's Agency for Health and Food Safety (AGES) found a series of ethical and legal misdemeanours, which led them to conclude that the trial was illegal and invalid (see Nature 454, 922-923; 2008). The Lancet withdrew Strasser's paper reporting the trial's results (S. Kleinert and R. Horton Lancet 372, 789-790; 2008).

But on 8 September the Vienna-based National Disciplinary Committee - which adjudicates on employment issues relating to civil servants (including university professors) - revoked Strasser's suspension. The committee based its decision on the outcome of a case brought to an Innsbruck court in which the university hospital sued Strasser and his department head Georg Bartsch for $€ 1.2$ million (US\$1.6 million) - its estimated cost for giving Strasser's treatment to 400 patients not involved in clinical trials. The court refused the claim on 3 August, stating that there was no proof that Strasser had intended financial deception. Bartsch was too ill to stand trial.
However, the court also stated that Strasser had provided false testimony during a 2008 civil damages case brought by a patient who had received the treatment, and that he had falsified evidence in the AGES investigation, which considered legal issues surrounding the clinical trials. It fined him $€ 4,500$.

The university plans to appeal against Strasser's reinstatement. In the meantime, the university has asked Strasser only to prepare unscheduled lectures: "We don't want him to get into clinical work," says Lochs.

In separate legal cases, several patients who say the treatment harmed them are now trying to bring charges of grievous bodily harm against Strasser, according to the Innsbruckbased lawyer Thomas Juen, who has previously represented trial patients seeking damages.

Earlier this year, the Medical University of Innsbruck completed its own investigation into the scientific aspects of the case, finding that Bartsch and Strasser had engaged in what Lochs views as "massive scientific misconduct".

The university's slowness in carrying out the investigation has been widely criticized. One academic, who asked to remain anonymous, said he believed that a timely, formal statement that Strasser had perpetrated serious scientific misconduct might have helped avert the disciplinary committee's revocation of his dismissal. Juen adds that he is surprised that the disciplinary committee revoked Strasser's suspension so quickly, "given that appeals are ongoing, and that looming cases of grievous bodily harm on the part of patients have not yet come to court".

Strasser did not respond to Nature's request for an interview, and Bartsch was unavailable for comment as a result of his illness. Strasser, however, was quoted in a local newspaper as saying that he wants to return to clinical practice and that the hospital administration may not be able to stop him. .

\section{CORRECTION}

The News story 'Canadian ozone network faces axe' (Nature 477, 257-258; 2011) stated that Environment Canada planned to cut 776 jobs. Although 776 employees will be affected by workforce changes, only about 300 posts are being eliminated. 